\title{
Separating past and future autobiographical events in memory: Evidence for a reality monitoring asymmetry
}

\author{
Ian M. McDonough And David A. Gallo \\ University of Chicago, Chicago, Illinois
}

\begin{abstract}
After thinking about the past and imagining the future, how do people separate these real and imagined events in memory? We had subjects engage in past and future autobiographical elaboration, then later take memory tests that required them to recollect these earlier generated events. In Experiment 1, testing memory for previously generated past or future autobiographical events led to fewer source memory confusions than did an elaborative control task, suggesting that the distinctive features of autobiographical elaboration improved subsequent retrieval monitoring accuracy. In Experiment 2, we directly compared retrieval monitoring accuracy for previously generated past and future autobiographical events and found that subjects made fewer source confusions when searching memory for future events. This asymmetry suggests that the features characterizing future elaborations (e.g., cognitive operations) were used more effectively during reality monitoring than were the features characterizing past elaborations (e.g., perceptual details), and has implications for future-oriented theories of memory.
\end{abstract}

Memory confusion can occur between events that actually happened and events that were only imagined. For instance, people can confuse whether they performed some action or imagined it (e.g., Goff \& Roediger, 1998) or whether some autobiographical event was real or only imagined (e.g., Garry, Manning, Loftus, \& Sherman, 1996). Such confusions between an event originating from an external source (perception) and an event originating from an internal source (imagination) are called reality monitoring errors (see Johnson \& Raye, 1981, for a review). Reality monitoring fits into a general source monitoring framework (Johnson, Hashtroudi, \& Lindsay, 1993), asserting that people recollect features of information and must use these features of information to reconstruct memories. Because this reconstruction process is not perfect, we must infer missing features of information through retrieval monitoring processes.

To apply reality monitoring research to autobiographical memories, it is important to know what types of imagination are common or important in daily life and thereby most likely to be confused with real events. According to the constructive episodic simulation hypothesis, a critical function of autobiographical memory is to imagine possible future events and plans (Addis, Wong, \& Schacter, 2007; Schacter \& Addis, 2007; Schacter, Addis, \& Buckner, 2007). In fact, it has been argued that the ability to remember evolved from the need to generate plans and actions for the future (e.g., Suddendorf \& Busby, 2005; Suddendorf \& Corballis, 1997, 2007) and that the abil- ity to generate future simulations may have been given adaptive priority (Schacter et al., 2007). Consistent with the idea that past and future autobiographical elaboration have overlapping cognitive processes, recent studies have shown that the neural signatures of remembering the past (past elaboration) are similar to those of imagining the future (future elaboration) (e.g., Addis et al., 2007; Szpunar, Watson, \& McDermott, 2007), suggesting overlapping processes between past and future events. Moreover, like past elaboration, future elaboration entails the creation of a scene that has a narrative structure accompanied by a sense of mental time travel (Tulving, 1985) and is likely to involve self-referential processing when considering one's future goals or plans (Conway, 2005). This research highlights the similarities between past and future events, in that both involve reconstructive processes that operate on similar features or episodic content (Botzung, Denkova, \& Manning, 2008; Buckner \& Carroll, 2007; Schacter et al., 2007; Szpunar et al., 2007).

If imagination of future autobiographical events is a critical function of human memory, the high degree of overlap between past memories and future imaginations that has recently been uncovered by neuroimaging and subjective reports poses a major theoretical question for reality monitoring processes. Because they share so many cognitive and neural processes, how are we able to effectively discriminate between the two in our daily lives? How do we avoid constantly confusing the past and the future?

I. M. McDonough, imcdono@uchicago.edu 
One clue to this question comes from studies in which the subjective features of real and imagined events, such as dreams, fantasies, or unfulfilled intentions, have been compared (e.g., Hassabis, Kumaran, \& Maguire, 2007; Johnson, Foley, Suengas, \& Raye, 1988; Johnson \& Raye, 1981; Kealy \& Arbuthnott, 2003; McGinnis \& Roberts, 1996). In each of these studies, subjects were asked to retrieve memories for past or imagined autobiographical events and then to rate these memories on numerous subjective features. The findings from these studies consistently demonstrate that memories for past autobiographical events were rated as having more perceptual details, whereas memories for imaginations were rated as containing more details about one's thoughts (i.e., cognitive operations; Johnson \& Raye, 1981). Imaginations are thought to contain more cognitive operations, because more effort is needed to construct an imagination than to recall a past event. Although these researchers did not directly consider the imagination of future events, their results are in line with more recent studies in which past and future autobiographical elaborations were compared. For instance, past autobiographical elaborations have consistently been shown to contain more perceptual features than future events (e.g., Berntsen \& Jacobsen, 2008; D’Argembeau \& Van der Linden, 2004; Szpunar \& McDermott, 2008). In addition, fMRI studies have shown that future elaboration reliably produces more activation in the frontal lobes than past elaboration, which may reflect the additional cognitive operations required to construct simulations of new events (e.g., Addis et al., 2007). However, in at least one other study (Botzung et al., 2008), increased activation in prefrontal regions for past relative to future events has been found, highlighting the need for additional behavioral experiments to tease apart differences in how we process past and future autobiographical events.

Most researchers investigating reality monitoring have used simple stimuli (e.g., viewing and imagining pictures of objects) with atemporal imaginations, so their generality toward understanding reality monitoring for past or future events is questionable. For instance, the neural signatures and the subjective memorial features used to accurately discriminate previously seen objects and previously imagined objects differ from features used to discriminate between memories and imaginations of autobiographical events (Hassabis et al., 2007). Furthermore, out of all of the researchers who have investigated the subjective features that differentiate past and future autobiographical events, none has attempted to determine whether these subjective differences actually have consequences for subsequent reality monitoring accuracy. The present study was aimed at this last question.

In the present study, we investigated the influence of past and future event elaboration on objective memory accuracy. To do this, we developed a subsequent memory procedure. With this procedure, subjects first generated past and future events and rated the events' subjective features (as in prior work). The subjects then took a memory test that allowed us to objectively measure their ability to discriminate between these previously generated events. In this way, we were able to test the degree to which sub- jective differences observed during event generation actually tracked subsequent retrieval monitoring accuracy. This procedure allowed us to investigate which of these various features may be most important for reality monitoring and, in particular, how they might contribute to a retrieval monitoring process called the distinctiveness heuristic (Schacter, Israel, \& Racine, 1999).

\section{The Distinctiveness Heuristic}

The distinctiveness heuristic is a process that enhances retrieval monitoring. This heuristic is used when a person expects to recollect detailed features for a class of events and, in the absence of such features, infers that a questionable event must not have occurred (Schacter et al., 1999; see Schacter \& Wiseman, 2006, for a review; also see Strack \& Bless, 1994, for similar ideas). For example, subjects can use perceptual features found in pictures as diagnostic criteria to help distinguish between items that were previously viewed as pictures and those that were not. When items that were not studied as pictures fail to meet these criteria on subsequent recognition tests or source memory tests, the subjects can correctly reject that item as having occurred, thereby avoiding memory distortions. The distinctiveness heuristic is more generally considered a type of diagnostic monitoring process that extends beyond pictorial encoding, including semantic processing (Gallo, Meadow, Johnson, \& Foster, 2008), imagination (Foley, Wozniak, \& Gillum, 2006; Wiseman, 2007), and anagram generation (Gunter, Bodner, \& Azad, 2007).

In a recent study, McDonough and Gallo (2008) extended the distinctiveness heuristic to autobiographical elaboration, or the retrieval of a specific autobiographical memory associated with a cue word. Subjects made fewer source memory confusions when searching memory for previous autobiographical elaborations (i.e., "Did you generate an autobiographical memory for this test word earlier?") relative to semantic control tasks (i.e., "Did you make a semantic judgment for this test word earlier?"). Moreover, these effects were dissociated from true recognition by manipulating the relative familiarity of the autobiographical and semantic items (via study repetition). These and other results in the literature bolster the idea that autobiographical elaboration leads to more distinctive recollections than do other deep levels of processing (cf. Craik \& Tulving, 1975; Greenwald \& Banaji, 1989), thereby facilitating recollection-based monitoring processes.

McDonough and Gallo (2008) demonstrated that the subsequent memory test procedure can provide insights into autobiographical memory monitoring, but that study did not include a future autobiographical elaboration task. In the present study, the methods used by McDonough and Gallo were adapted to include both past and future autobiographical elaboration. In Experiment 1, we set out to replicate the findings of McDonough and Gallo for past events, using a more elaborate control task, and also to determine whether retrieval monitoring is enhanced for future events. To anticipate, study ratings confirmed that both past and future elaborations contained distinctive features relative to the semantic control, and these features 
enhanced retrieval monitoring on subsequent tests. On the basis of these findings, Experiment 2 was designed to directly contrast past and future elaboration to determine which features might be more important in making reality monitoring decisions.

\section{EXPERIMENT 1}

The goal of Experiment 1 was to determine which features of autobiographical elaborations make them distinctive and whether these features are specific to the generation of episodic-like events (i.e., events containing unique features such as self-referential processing, a narrative structure, rich contextual details, and a sense of mental time travel). McDonough and Gallo (2008) found that autobiographical elaboration for past events was associated with fewer subsequent memory confusions than were semantic control tasks, but these effects may have been due to features that are specific to episodic-like events or to greater cognitive elaboration in general. Experiment 1 was designed to test these ideas by comparing autobiographical elaboration to a sentence generation task that was designed to involve more cognitive elaboration than the previously used semantic judgments. We also compared two types of autobiographical elaboration: one based on actual past events (as in McDonough \& Gallo, 2008) and one based on imagined future events (as in Addis et al., 2007). These two tasks were thought to elicit a similar degree of episodic-like elaboration, but to differ in the amount of corresponding details (as well as in whether or not the event was real) that may differentially contribute to reality monitoring. Following the encoding tasks, memory accuracy was measured via criterial recollection tests (Gallo, Weiss, \& Schacter, 2004). These tests were designed to assess differences in how people monitor their memory as a function of their retrieval orientation (i.e., selectively searching memory for past, future, or sentence elaborations).

Our primary interest on the subsequent memory tests was in false alarm rates to items studied in a fourth task (definition generation), which reflect source confusions. The definition task was designed to provide a degree of semantic elaboration similar to that of the other tasks but differed from these other tasks in that events or objects did not need to be generated and linked to the cue word. Because these definition items had the same studied history across all three tests, different levels of source confusion would reflect the differences in retrieval orientation across test blocks. If the episodic-like nature of autobiographical elaboration is the critical factor, with no additional impact of the more fine-grained features that may distinguish past and future events, we might expect past and future elaboration to elicit the same accuracy benefit relative to sentence generation. In contrast, if episodic details specific to past events (e.g., perceptual details) are most critical, we would expect the greatest accuracy when we test for past events (i.e., those that actually occurred), because they have been found to contain more perceptual details than imagined future events. Finally, if the degree of semantic elaboration (e.g., generation, semantic integration, and item-specific processing) is the most critical factor, with no additional benefit of the generation of autobiographical or episodic-like events, we would expect similar levels of accuracy across the three test blocks.

\section{Method}

Subjects. Sixty students (44 female) at the University of Chicago participated in the experiment for course credit or $\$ 15$. The data from 11 subjects failed to record, resulting in a total of 49 subjects.

Materials and Design. The stimuli included 96 common object words used in McDonough and Gallo (2008). At study, the subjects received four tasks: past elaboration, future elaboration, sentence generation, and definition generation. In the past, future, and sentence tasks, the subjects studied 16 different object words. Eight of those object words were studied only in the assigned task, whereas the other 8 occurred in both the sentence and definition tasks (both items). Additionally, 24 words were studied only in the definition task (excluding both items), and 24 words were never studied. Words were counterbalanced across the eight types of items (past only, future only, sentence only, definition only, both past and definition, both future and definition, both sentence and definition, and nonstudied). Each encoding task was counterbalanced across subjects, with the exception that the definition task (which was used to generate studied lures for the subsequent tests) always occurred first. Generated events in each task were typed and recorded along with each subject's ratings of each task.

As in the previous experiments in which the criterial recollection task was used, both items were included at study so that the sources were not mutually exclusive. This control ensured that the subjects had to selectively search their memory for the criterial recollection, as opposed to recalling one source for an item and inferring that the other source could not have occurred (i.e., a recall-to-reject strategy; see Gallo, Cotel, Moore, \& Schacter, 2007).

The next day, the subjects were given three tests (past test, future test, and sentence test) with order counterbalanced across subjects. The subjects were presented with each word in the same font as that in the study phase, along with the appropriate test prompt (i.e., "Did you retrieve a specific memory for this item?" during the past test, "Did you imagine a future event for this item?" during the future test, and "Did you create a sentence for this item?" during the sentence test). The tests consisted of 32 items (randomly arranged) consisting of 8 items of each type (targets, studied lures, both items, nonstudied lures). Targets were items that corresponded to the appropriate test (e.g., past items on the past test), studied lures were items that only occurred in the definition task, and both items were those that the subjects studied in the target task and the definition task (also targets).

Procedure. For all tasks, elaborations were generated using the Galton-Crovitz word-cuing technique (Galton, 1879; Crovitz \& Schiffman, 1974). The subjects were first presented with the definition generation task, in which the subjects viewed an object word and were asked to write a one-sentence definition of the object. In the past elaboration task, the subjects were asked to retrieve a different specific memory associated with each item and to type one sentence describing what the memory involved and where it took place. They were asked to exclude private details that they felt uncomfortable recording. In the future elaboration task, the subjects were asked to imagine a different specific future event associated with each item and to type one sentence describing what the event would involve and where it would take place. The subjects were asked to create a new event rather than to recount an actual memory or any part of one. In the sentence generation task, the subjects were asked to generate a related object and to type one sentence describing how the two items were functionally related (see Addis et al., 2007, and Greenwald \& Banaji, 1989, for a similar semantic comparison task). For instance, 1 subject was cued with orange; generated juice; and typed, "Juice can be made from oranges."

For each task, an object word was presented in the middle of the computer screen in black text, with a prompt reminding them of the task (e.g., "Retrieve a specific memory"). The object word appeared 
Table 1

Mean Study Ratings As a Function of Item Type in Experiment 1

\begin{tabular}{|c|c|c|c|c|c|c|c|c|}
\hline \multirow[b]{3}{*}{ Item Type } & \multicolumn{8}{|c|}{ Rating Type } \\
\hline & \multicolumn{2}{|c|}{ Difficulty } & \multicolumn{2}{|c|}{$\begin{array}{l}\text { Perceptual } \\
\text { Details }\end{array}$} & \multicolumn{2}{|c|}{$\begin{array}{c}\text { Emotional } \\
\text { Arousal }\end{array}$} & \multicolumn{2}{|c|}{ Uniqueness } \\
\hline & $M$ & $S E M$ & $M$ & $S E M$ & $M$ & $S E M$ & $M$ & $S E M$ \\
\hline Past elaboration & 2.78 & .14 & 3.88 & .18 & 3.66 & .17 & 3.77 & .16 \\
\hline Future elaboration & 3.03 & .19 & 3.62 & .18 & 3.31 & .18 & 3.39 & .16 \\
\hline Sentence generation & 2.88 & .18 & 3.39 & .18 & 2.86 & .19 & 3.08 & .18 \\
\hline Definition generation & 3.33 & .16 & 3.67 & .15 & 2.53 & .15 & 3.23 & .17 \\
\hline
\end{tabular}

Note-All ratings were made on a scale of 1 (low) to 7 (high).

for only $5 \mathrm{sec}$, but the subjects were given a total of $30 \mathrm{sec}$ to record their descriptions before the task ratings appeared, similar to what was done in prior studies in which autobiographical memories were investigated (Conway, 2005; Wenzel, 2005). If the subjects finished recording their responses within the $30 \mathrm{sec}$, they could press the "return" key to continue. After recording each description, the subjects rated each generated item on four features on a scale from 1 (low) to 7 (high): task difficulty (as a proxy for cognitive operations), perceptual details, emotional arousal, and uniqueness (self-paced).

The next day, the subjects returned and took three criterial recollection tests: past, future, and sentence. On the past test, the subjects were told that the test included past only, definition only, both, and new items. They were instructed to press the "yes" key if they remembered retrieving a specific memory associated with the item (past only or both items) and otherwise to respond with the "no" key (definition only or new items). They were also told that remembering whether they constructed a definition for the item was irrelevant on this test, because, for some items, they both retrieved a specific memory and typed a definition for the item. Therefore, they had to selectively search their memory to determine whether they had retrieved a specific memory associated with the item earlier. Analogous instructions were given for the future and sentence tests, with the only change being in the to-be-recollected (or criterial) information.

\section{Results and Discussion}

Corresponding effect sizes were calculated using Cohen's $d$ for $t$ tests. Unless otherwise specified, each significance test used a two-tailed distribution $(p<.05)$.

Study ratings. Study ratings can be seen in Table 1 . We first compared past and future elaboration ratings with sentence ratings to confirm that episodic content would enhance the number of generated features specific to each encoding task. Relative to the semantic control task $(M=$ 3.39), past elaboration elicited memories that contained more perceptual details $(M=3.88)[t(48)=4.56, p<.001$, $S E M=.109, d=.40]$, were more arousing $(M=3.66 \mathrm{vs}$. $M=2.86)[t(48)=6.36, p<.001, S E M=.127, d=.64]$, and were more unique $(M=3.77$ vs. $M=3.08)[t(48)=$ $4.98, p<.001, S E M=.139, d=.57]$. Similarly, relative to the semantic control task, future elaboration elicited events that contained marginally more perceptual details $(M=$ 3.62) $[t(48)=1.791, p=.08, S E M=.127, d=.18]$, were more arousing $(M=3.31)[t(48)=3.82, p<.001, S E M=$ $.120, d=.35]$, and were more unique $(M=3.39)[t(48)=$ $2.19, p=.034$, SEM $=.139, d=.25]$.

We also found differences between past and future events that were consistent with the results of prior work (e.g., D'Argembeau \& Van der Linden, 2004). Past elaboration elicited memories that were rated as containing more details $[t(48)=2.08, p=.043, S E M=.123, d=$
$.21]$, were more arousing $[t(48)=2.90, p=.006$, SEM $=$ $.121, d=.29]$, and were more unique $[t(48)=3.32, p=$ $.002, S E M=.118, d=.34]$ than future elaboration. However, future elaboration elicited events that were rated as more difficult to generate (i.e., required more cognitive operations) than past elaboration $(M=2.78)[t(48)=$ $-1.84, p=.036, S E M=.134, d=.21$, one-tailed] (see Hassabis et al., 2007; Johnson et al., 1988).

Criterial recollection test. The test phase results can be found in Table 2. The same general pattern was observed on each of the three tests, a result consistent with the results of prior work in which the criterial recollection task was used with other types of materials (e.g., Gallo et al., 2004; McDonough \& Gallo, 2008). First, hits to both targets were greater than hits to studied targets, because the former were presented twice during the study phase (all $p$ s $<.05$ ). Second, hits to studied targets were greater than false alarms to studied lures (definition items), demonstrating that the subjects were able to make the appropriate source discrimination on each test (all $p \mathrm{~s}<.001$ ). Finally, false alarms to studied lures were greater than false alarms to nonstudied lures, demonstrating source confusions (all $p \mathrm{~s}<.01$ ).

The critical comparison was of the levels of false alarms to the studied lures across the three tests. As was predicted, false alarms to studied lures on the past test were lower than those on the sentence test $[t(48)=-2.00, p=$ $.026, S E M=.034, d=.23$, one-tailed] (see McDonough $\&$ Gallo, 2008). False alarms to studied lures on the future test also were lower than false alarms to studied lures on

Table 2

Mean Proportion Recognized As a Function of Test Type in Experiment 1

\begin{tabular}{lll}
\hline Test Type & $M$ & SEM \\
\hline Past Test & & \\
Both hit & .80 & .03 \\
Past hit & .69 & .03 \\
Definition FA & .16 & .04 \\
New FA & .03 & .02 \\
Future Test & & \\
Both hit & .73 & .03 \\
Future hit & .65 & .03 \\
Definition FA & .12 & .03 \\
New FA & .03 & .02 \\
Sentence Test & & \\
Both hit & .77 & .03 \\
Sentence hit & .69 & .03 \\
Definition FA & .23 & .04 \\
New FA & .04 & .02 \\
\hline
\end{tabular}

Note-FA, false alarm. 
the sentence test $[t(48)=-2.59, p=.013, S E M=.042$, $d=.41]$. Thus, the subjects selectively reduced their false alarms to studied lures on the past and future tests relative to those on the sentence test. In contrast, false alarms to studied lures on the past test were equal to the false alarms to studied lures on the future test $(p=.23)$.

To the extent that false alarm differences in this task reflect the use of a distinctiveness heuristic, these results suggest that the items processed via past elaboration were as distinctive as the items processed via future elaboration, but each of these processes was more distinctive than elaborative semantic processing. These findings are consistent with the idea that autobiographical elaboration is not distinctive solely because of semantic elaboration; episodiclike qualities also contribute. Past and future elaboration should have involved more self-referential processing, narrative structure, and a sense of mental time travel than the sentence task. Furthermore, these findings suggest that the episodic-like qualities of autobiographical elaboration do not need to correspond to real events to elicit a distinctiveness heuristic, because never-experienced future events elicited the same retrieval monitoring benefit.

The subjective ratings were consistent with these conclusions. Compared with the semantic task, past and future elaboration elicited events that were more perceptually detailed, arousing, and unique. These various features likely contributed to the distinctiveness of generating episodiclike events. However, direct comparisons between subjective ratings of past and future elaboration suggested that past events were more perceptually detailed, arousing, and unique than future events, whereas future events were more difficult to generate than past events. Thus, despite finding similarities when compared with the semantic control task, past and future elaboration may be distinctive for different reasons. These differences may help discriminate between past and future events when they are directly compared in memory, as might happen in more common reality monitoring situations. Experiment 2 was designed to further investigate this idea.

\section{EXPERIMENT 2}

To better understand which features differentiate past and future events in reality monitoring situations, we had subjects in Experiment 2 directly discriminate between items processed via past and future elaboration. According to the source monitoring framework, the criteria that subjects use to make memory decisions should change depending on the types of items being discriminated. Thus, whereas features such as self-referential processing, records containing a narrative structure, and the sense of mental time travel may have helped to discriminate autobiographical events from definition items in Experiment 1, these same features should be less helpful in discriminating between past and future autobiographical events in Experiment 2. These features are less diagnostic of the occurrence of either event, because they are contained in both past and future events.

To the extent that distinctive features among a class of items lead to enhanced retrieval monitoring, we should find differences in false alarm rates across the past and future tests. For instance, if subjects expect perceptual details in past events to have stronger or more unique recollections than future events, they should weight these features more heavily when searching memory for past events. In contrast, if the subjects expect cognitive operations in future events to have stronger or more unique recollections than past events, they should weight these features more heavily when searching memory for future events. Any accuracy differences between the past and future tests should reflect differences in these retrieval expectations, with the possibility that one type of feature more accurately contributes to reality monitoring than the other type.

Existing perspectives regarding the primary function of memory make competing predictions as to which types of features (past or future) would be remembered more accurately. Intuitively, the primary function of memory seems to be to reconstruct the past. From this perspective, one might expect past events to have richer features than future events. Thus, subjects would more accurately monitor memory when oriented toward the past elaboration task (i.e., when on the past test). This prediction is consistent with the previously mentioned work showing that the actual features of perceived events tend to be greater than imagined future events (e.g., Berntsen \& Jacobsen, 2008; D'Argembeau \& Van der Linden, 2004; Szpunar \& McDermott, 2008). It is also consistent with false memory work suggesting that memories for actually experienced events have more recollected details than memories for events that did not actually occur (e.g., Mather, Henkel, \& Johnson, 1997; Norman \& Schacter, 1997).

In contrast to this emphasis on retrospection, some researchers have argued that the primary function of memory is to imagine and plan for future events (e.g., Addis et al., 2007; Schacter et al., 2007; Suddendorf \& Busby, 2005; Suddendorf \& Corballis, 1997, 2007). From this perspective, it is important to have a strong memory for a previously imagined future event, so that one later remembers that it has not been done and that one still has to complete the intended action. Intended actions would be meaningless if we planned them but never carried them through because we thought that they had already occurred. To the degree that future-oriented planning and behaviors are a critical function of memory, one might predict more accurate memory for previously generated future events. By this account, subjects might be more accurate at monitoring memory when retrieval is oriented toward the future elaboration task (i.e., on the future test).

\section{Method}

Subjects. Fifty-five students (34 female) at the University of Chicago participated in the experiment for course credit or $\$ 15$. Seven subjects were removed from the data analyses, because they received the wrong experimental scripts (4 subjects) or did not return for the second day of testing ( 3 subjects), resulting in a total of 48 subjects.

Materials and Design. The stimuli included a subset of the common object words from Experiment 1. At study, the subjects received 18 past elaboration items, 18 future elaboration items, and 18 items in both tasks, with 18 items remaining unstudied (counterbalanced 
across subjects). As in the previous experiment, both items were included at study to prevent the subjects from using an exclusion strategy at test. The subjects typed each elaboration and then made subjective ratings.

The test phase occurred the next day and consisted of two tests (a past and a future test) with order counterbalanced across subjects. The past and future tests were the same as those in Experiment 1, with a few exceptions. During each test, the subjects were presented with 36 items (randomly arranged) consisting of 9 items of each type (targets, studied lures, both items, nonstudied lures). Unlike those in Experiment 1, studied lures were future elaboration items on the past test and past elaboration items on the future test.

Procedure. The subjects were given four study blocks alternating between the past and future elaboration conditions (block order counterbalanced across subjects). The procedure for each task was identical to that in Experiment 1, with the exception that the subjects had to rate an additional three features: valence (1, negative; 7 , positive), temporal distance (1, recent; 7 , distant $)$, and importance $(1$, not important; 7 , very important).

The next day, the subjects returned and took two criterial recollection tests: past and future. On the past test, the subjects were told that the test included past only, future only, both, and new items. They were instructed to press the "yes" key if they remembered retrieving a specific memory associated with the item (past only or both items), otherwise to respond with the "no" key (future only or new items). They were also told that remembering whether they had imagined a future event for the item was irrelevant on this test, because for some items, they both retrieved a specific memory and imagined a future event. Analogous instructions were given for the future test, with the only change being in the to-be-recollected (or criterial) information. If the subjects responded yes on either test, they were also asked to rate the unique recollected details, or distinctiveness, of each item on a scale from 1 to 7 (see the Appendix). This rating was designed to provide a more detailed measure of qualitative recollection differences assumed by the distinctiveness heuristic hypothesis.

\section{Results and Discussion}

Study ratings. Study ratings can be seen in Table 3 . The subjects rated perceptual details higher for past events $(M=4.33)$ than for future events $(M=3.85)[t(47)=$ 4.56, $p<.001, S E M=.106, d=.52]$ but rated it more difficult to generate future events $(M=2.78)$ than past events $(M=2.48)[t(47)=-3.36, p=.002, S E M=$ $.090, d=.39]$. These differences between past and future events were consistent with the results of Experiment 1 and also with those of other studies in which real and imagined events were compared (e.g., Johnson \& Raye, 1981; Johnson, Raye, Foley, \& Foley, 1981). Future events $(M=3.50)$ were rated as more important than past events $(M=3.20)[t(47)=-3.56, p=.001, S E M=.084, d=$

Table 3

Mean Study Ratings As a Function of Item Type in Experiment 2

\begin{tabular}{|c|c|c|c|c|}
\hline \multirow[b]{3}{*}{ Rating Type } & \multicolumn{4}{|c|}{ Item Type } \\
\hline & \multicolumn{2}{|c|}{$\begin{array}{c}\text { Past } \\
\text { Elaboration }\end{array}$} & \multicolumn{2}{|c|}{$\begin{array}{c}\text { Future } \\
\text { Elaboration }\end{array}$} \\
\hline & $M$ & SEM & $M$ & $\overline{S E M}$ \\
\hline Difficulty & 2.48 & .10 & 2.78 & .12 \\
\hline Perceptual details & 4.33 & .13 & 3.85 & .14 \\
\hline Emotional arousal & 3.78 & .13 & 3.87 & .13 \\
\hline Uniqueness & 3.79 & .13 & 3.74 & .14 \\
\hline Valence & 4.48 & .08 & 4.75 & .08 \\
\hline Recency & 4.64 & .13 & 4.25 & .13 \\
\hline Importance & 3.20 & .11 & 3.50 & .12 \\
\hline
\end{tabular}

Note-All ratings were made on a scale of 1 (low) to 7 (high).
.38], more positive than past events $(M=4.75$ and 4.48 for future and past events, respectively) $[t(47)=-4.66$, $p<.001, S E M=.057, d=.48]$, and more recent than past events $(M=4.25$ and 4.64 for future and past events, respectively) $[t(47)=2.97, p=.005, S E M=.133, d=$ .44]. These differences are also consistent with the results of prior research (e.g., Addis et al., 2007; Berntsen \& Jacobsen, 2008; D’Argembeau \& Van der Linden, 2004).

Unlike in Experiment 1, we found that the subjects' ratings for arousal did not differ between past $(M=3.78)$ and future $(M=3.87)$ events $(p=.22)$, nor did uniqueness ratings between past $(M=3.79)$ and future $(M=$ $3.74)$ events $(p=.63)$. Some researchers have found emotional arousal to be rated higher for future events than for past events (e.g., D'Argembeau \& Van der Linden, 2004), whereas others have found no differences (e.g., Addis et al., 2007; Berntsen \& Jacobsen, 2008). These mixed findings suggest that these subjective ratings do not consistently characterize past or future events and may not be diagnostic of either past or future events (for a similar discussion regarding emotion and atemporal imaginations, see Johnson, 2006).

Because this experiment involved a direct comparison between past and future autobiographical events, we also analyzed how often the subjects may have recast past events into the future. Recasting is when subjects recall a recent autobiographical event and imagine that such an event may occur in the next few weeks (Addis, Pan, Vu, Laiser, \& Schacter, 2009). To investigate the amount of recasting, we rated items for which the subjects generated both a past and a future event (both items). These items provided the strongest test of recasting, because the subjects had previously seen an object word corresponding to either the past or the future elaboration instructions. Thus, they may be more inclined to remember what they generated previously and to recast the event into the alternative time period. For each pair of generated items, the first author (I.M.) rated the degree of overlap as having no overlap, partial overlap, or full overlap. Pairs with partial overlap were items that had some degree of similarity but could have been describing different events. For example, when given the cue spider on the past elaboration task, one subject typed, "I saw a spider in the woods," and on the future elaboration task the subject typed, "I will see a spider when I camp this summer." Pairs with full overlap were items that either had no differences in the description or had only a difference of time period. For example, when given the cue mushroom on the past elaboration task, one subject typed, "I had Lithuanian mushrooms last Thanksgiving," and on the future elaboration task, the subject typed, "I will have Lithuanian mushrooms at Thanksgiving."

Although some recasting may have occurred, it did not occur for most of the both items, confirming that the subjects had followed the instructions and generated unique events for each cue. Out of 18 pairs of items, a mean of $14.88(S D=2.56)$ were rated as having no overlap, 1.44 $(S D=1.25)$ as having partial overlap, and $1.38(S D=$ $1.70)$ as having full overlap. The amount of recasting for any degree of overlap did not depend on whether the subjects generated past or future events first. 
Criterial recollection test. The test results can be found in Table 4. As in Experiment 1, the same general pattern was observed on each of the two tests. First, hits to both items were greater than hits to studied targets, although this effect was only significant on the past test ( $p=.002$; future test, $p=.08$ ). Second, hits to studied targets were greater than false alarms to studied lures, demonstrating that the subjects were able to make the appropriate source discrimination on each test (both $p \mathrm{~s}<.001$ ). Finally, false alarms to studied lures were greater than false alarms to nonstudied lures, demonstrating source confusion (both $p \mathrm{~s}<.001)$.

Most important, there were fewer false alarms to studied lures on the future test than on the past test $[t(47)=$ $2.21, p=.032, S E M=.042, d=.39]$. This finding indicates a reality monitoring asymmetry: The subjects were more likely to make memory confusions when searching memory for past events than when searching memory for future events. This asymmetry suggests that at least some of the features in memory that were stronger for future events (e.g., cognitive operations, importance, positivity, and recentness) contributed more to retrieval monitoring accuracy than those features that were stronger for past events (e.g., perceptual details and negativity).

Finally, we compared the ratings of unique recollected details obtained on the past and future tests. This analysis revealed that there were no differences between ratings for past and future hits $(p=.71)$ to studied targets or false alarms $(p=.79)$ to studied lures. Coupled with the subjective ratings obtained at study, these test ratings suggest that past and future events were equally distinctive, but for different reasons. They also suggest that the greater accuracy that we observed on the future test than on the past test is not due to overall differences in recollective distinctiveness across these types of events. Instead, this reality monitoring asymmetry stemmed from the different weighting of features that the subjects used to monitor retrieval for each type of event on each test. In other words, although the amount of unique features are the same in past and future events, the subjects may have been more confident when recalling the features characteristic of future events than when recalling those of past events, weighting these features more heavily and leading to a greater accuracy.

We also compared unique recollected details for correct and incorrect items on each test. Unique recollected

Table 4

Mean Proportion Recognized As a Function of Test Type in Experiment 2

\begin{tabular}{ccc}
\hline Test Type & $M$ & SEM \\
\hline Past Test & & \\
Both hit & .85 & .02 \\
Past hit & .74 & .03 \\
Future FA & .27 & .04 \\
New FA & .04 & .02 \\
Future Test & & \\
Both hit & .78 & .03 \\
Future hit & .71 & .03 \\
Past FA & .18 & .02 \\
New FA & .04 & .02 \\
\hline
\end{tabular}

Note-FA, false alarm. details for correctly recognized targets were greater than those to falsely recognized lures $(M=4.42$ and 3.28 on the past test, and $M=4.37$ and 3.18 on the future test; all $p \mathrm{~s}<.001)$. This finding indicates that true recognition was associated with more unique or distinctive recollections than source confusions, a finding consistent with false memory tasks (e.g., Mather et al., 1997; Norman \& Schacter, 1997).

\section{GENERAL DISCUSSION}

In the present study, we provided several new insights into the features that make autobiographical elaboration distinctive, in general, and also those features that are used to separate past from future autobiographical events in memory. In Experiment 1, we investigated the extent to which autobiographical elaboration elicited distinctive processing relative to semantic elaboration. We found that source confusion errors were reduced when searching memory for both past and future elaborations relative to the semantic task. Because of our use of a more elaborate semantic control task than that used in McDonough and Gallo (2008), the present results more strongly indicate that the generation of episodic-like details during autobiographical elaboration is sufficient to facilitate retrieval monitoring, beyond any benefit arising from semantic elaboration and constructive processes.

We also extended our prior findings by observing a similar autobiographical elaboration benefit for both past and future events, suggesting that the encoding of episodic-like events but not necessarily real events was sufficient to enhance memorial distinctiveness. Consistent with this idea, several subjective features were greater for past and future events relative to the semantic control task (e.g., perceptual details, arousal, and uniqueness), although there were also some differences between past and future events.

Having established that past and future autobiographical elaborations are distinctive processes in Experiment 1, in Experiment 2 we investigated how past and future events are discriminated from each other in memory. This experiment revealed a reality monitoring asymmetry. Across the two test blocks, the subjects were more likely to confuse these two events when selectively searching memory for previously generated past events than when searching memory for future events. Because these two types of tests differed only in retrieval orientation, this asymmetry indicates that the features that were stronger for future events contributed more to reality monitoring accuracy than did those features that were stronger for past events.

Why was accuracy greater when searching memory for future events than when searching for past events? One idea is that future events necessarily involve fragments of past memories (e.g., Szpunar \& McDermott, 2008), increasing the likelihood that subjects would confuse future events with past events on the past test. However, this process should have affected the past and future tests to the same extent in our procedure. By design, the two test blocks in Experiment 2 involved discriminating between the same types of past and future events. All other fac- 
tors being equal, any overlapping content between past and future events should have created an equal amount of confusion between these events on the tests. The only difference across our tests was in the retrieval orientation, so that the subjects searched memory for features that were more characteristic of past events on the past test and for features more characteristic of future events on the future test. Thus, the asymmetry that we observed must derive from the differential weighting of memorial features across the two tests, with features that were more characteristic of future events being more diagnostic than features that were more characteristic of past events.

Subjective ratings at study provided insights into the mechanisms underlying this reality monitoring asymmetry. Although the past events contained more perceptual details than future events, the future events contained more cognitive operations (as indexed by difficulty), consistent with the results of prior work (e.g., D'Argembeau \& Van der Linden, 2004). In addition, the future events were also rated as more positive, important, and recent than past events. These results are also consistent with those of the few studies in which differences in these features have been investigated (e.g., Berntsen \& Jacobsen, 2008; D'Argembeau \& Van der Linden, 2004). Furthermore, many researchers have found future events to be more important or personally significant than past events (e.g., Addis, Wong, \& Schacter, 2008; D’Argembeau \& Van der Linden, 2004). Consistent with the valence ratings in the present study, prior research has also suggested that people tend to be optimistic about future events (Sharot, Riccardi, Raio, \& Phelps, 2007). Any or all of these features may have served as diagnostic cues to facilitate monitoring accuracy on the future test relative to the past test.

Although, to our knowledge, we are the first to investigate how past and future events are discriminated on a subsequent memory task, there are several relevant links to prior work. One area is memory for experimentally performed actions. Many prior studies have shown that imagination can influence memory attributions when subjects search their memory for real past events, known as imagination inflation (e.g., Garry et al., 1996; Goff \& Roediger, 1998; Lampinen, Odegard, \& Bullington, 2003; Pezdek, Blandon-Gitlin, \& Gabbay, 2006; Thomas, Bulevich, \& Loftus, 2003). These studies have shown that the more times an event is imagined, the more likely people are to believe that the event actually happened, because perceptual details contained in the event increase. For instance, Lampinen et al. (2003) employed a source test for enacted events and imagined events accompanied by ratings for the qualitative features of each event. As subjects repeatedly imagined events, their qualitative features (details for thoughts, emotions, and kinesthetics) became more similar. At the same time, increased imagining led to more reality monitoring errors. These studies generally emphasize the weight that subjects give to perceptual details when making reality monitoring decisions.

Although it is likely that people do use perceptual details to monitor their memories, our findings suggest that perceptual details may not be the most critical feature when people engage in reality monitoring of autobiographical events. Because searching memory for future elaborations led to more accurate retrieval monitoring than did searching memory for past elaborations, cognitive operations rather than perceptual details were likely to be weighted more when making reality monitoring decisions.

A second related research area is prospective memory. Research on prospective memory tasks suggests that one critical function of memory is to keep track of important unfulfilled plans and intentions. Although people often have fleeting thoughts of actions that they need to carry out in the future, subsequent memory to carry out these actions is often poor without sufficient elaboration (Chasteen, Park, \& Schwarz, 2001). In contrast, when people develop elaborate mental images of the time at which and the place in which the future event is to occur (i.e., implementation intentions; Gollwitzer \& Brandstätter, 1997), subsequent prospective memory is drastically improved (Chasteen et al., 2001). Implementation intentions facilitate prospective memory performance, because people can take advantage of the rich features in these future imaginations.

Analogous to this prospective memory research, the present study suggests that people take advantage of the rich features in future imaginations when making reality monitoring decisions. Retrieval monitoring was enhanced when searching for imagined autobiographical events that were planned but had not yet occurred. Accurate memory for past events, although important, may not always be as important as accurate memory for previously imagined future events. For example, Spreng and Levine (2006) suggested that memory for future autobiographical events may be more important for human survival, enabling predictive control over one's environment, constraining behavior, and giving direction to one's actions. By imagining the future, we can play out alternative scenarios without suffering the consequences of each one, allowing the most beneficial action to be chosen (Suddendorf \& Corballis, 2007). However, this ability to play out alternative scenarios would be in vain if we then thought that we had already completed each action (i.e., confused future imagination with past occurrences). This idea is also consistent with the subjective ratings in the present study, indicating that the future events were more important and more recent (perhaps because they were more immediately relevant) than past events.

Of course, this functional interpretation may overemphasize the importance of future autobiographical imagination in daily life. An alternative explanation is that our future imagination task was more unique or unusual for the subjects (e.g., imagine an event that has never occurred before) than the retrieval of past autobiographical memories. Such a procedural difference may have resulted in more unique or unusual future events than past events, potentially enhancing reality monitoring for future events in Experiment 2. Although we cannot completely rule out this possibility, there was some evidence against it. Specifically, the ratings for the uniqueness of events generated at study and for the number of uniquely recollected details at test were not greater for future than for past items. To the contrary, we found that uniqueness rat- 
ings at study were greater for past items than for future items in Experiment 1, and the two were not significantly different in Experiment 2. In addition, ratings for unique recollected details did not differ for past and future hits in Experiment 2. Thus, although the future events did require more cognitive operations to generate than past events and were associated with other features that may have enhanced retrieval monitoring, the generated events themselves were not more unique or unusual than those in the past condition.

In summary, the present study demonstrates that past and future autobiographical elaborations lead to more accurate retrieval monitoring than a semantic elaboration task. This finding extends research on the distinctiveness heuristic to both past and future autobiographical events. Moreover, retrieval monitoring was more accurate for future elaborations than for past elaborations when the two were directly compared. This asymmetry suggests that people weight features characterizing future events more heavily than they do those characterizing past events in their memory decisions, potentially reflecting the importance of thinking about future autobiographical events.

\section{AUTHOR NOTE}

We thank Candace "Hope" Bias and Emily Graber for assistance collecting data. Aspects of these data were presented at the 80th Annual Meeting of the Midwestern Psychological Association (2008, Chicago, Illinois). Correspondence concerning this article should be sent to I. M. McDonough, Department of Psychology, University of Chicago, 5848 S. University Ave., Chicago, IL 60637 (e-mail: imcdono@uchicago.edu).

\section{REFERENCES}

Addis, D. R., Pan, L., Vu, M. A., Laiser, N., \& Schacter, D. L. (2009). Constructive episodic simulation of the future and the past: Distinct subsystems of a core brain network mediate imagining and remembering. Neuropsychologia, 47, 2222-2238. doi:10.1016/j .neuropsychologia.2008.10.026

Addis, D. R., Wong, A. T., \& Schacter, D. L. (2007). Remembering the past and imagining the future: Common and distinct neural substrates during event construction and elaboration. Neuropsychologia, 45, 1363-1377. doi:10.1016/j.neuropsychologia.2006.10.016

Addis, D. R., Wong, A. T., \& Schacter, D. L. (2008). Age-related changes in the episodic simulation of future events. Psychological Science, 19, 33-41. doi:10.1111/j.1467-9280.2008.02043.x

Berntsen, D., \& Jacobsen, A. S. (2008). Involuntary (spontaneous) mental time travel into the past and future. Consciousness \& Cognition, 17, 1093-1104. doi:10.1016/j.concog.2008.03.001

Botzung, A., Denkova, E., \& Manning, L. (2008). Experiencing past and future personal events: Functional neuroimaging evidence on the neural bases of mental time travel. Brain \& Cognition, 66, 202-212. doi:10.1016/j.bandc.2007.07.011

BucKner, R. L., \& CARroll, D. C. (2007). Self-projection and the brain. Trends in Cognitive Sciences, 11, 49-57. doi:10.1016/j.tics .2006.11.004

Chasteen, A. L., Park, D. C., \& Schwarz, N. (2001). Implementation intentions and facilitation of prospective memory. Psychological Science, 12, 457-461. doi:10.1111/1467-9280.00385

Conway, M. A. (2005). Memory and the self. Journal of Memory \& Language, 53, 594-628. doi:10.1016/j.jml.2005.08.005

Craik, F. I. M., \& Tulving, E. (1975). Depth of processing and retention of words in episodic memory. Journal of Experimental Psychology: General, 104, 268-294.

Crovitz, H. F., \& Schiffman, H. (1974). Frequency of episodic memories as a function of their age. Bulletin of the Psychonomic Society, 4, 517-518.

D'Argembeau, A., \& Van der Linden, M. (2004). Phenomenal characteristics associated with projecting oneself back into the past and forward into the future: Influence of valence and temporal distance. Consciousness \& Cognition, 13, 844-858. doi:10.1016/ j.concog.2004.07.007

Foley, M. A., WozNIAK, K. H., \& Gillum, A. (2006). Imagination and false memory inductions: Investigating the role of process, content and source of imaginations. Applied Cognitive Psychology, 20, 11191141. doi:10.1002/acp. 1265

Gallo, D. A., Cotel, S. C., Moore, C. D., \& Schacter, D. L. (2007). Aging can spare recollection-based retrieval monitoring: The importance of event distinctiveness. Psychology \& Aging, 22, 209-213. doi: 10.1037/0882-7974.22.1.209

Gallo, D. A., Meadow, N. G., Johnson, E. L., \& Foster, K. T. (2008), Deep levels of processing elicit a distinctiveness heuristic: Evidence from the criterial recollection task. Journal of Memory \& Language, 58, 1095-1111. doi:10.1016/j.jml.2007.12.001

Gallo, D. A., Weiss, J. A., \& Schacter, D. L. (2004). Reducing false recognition with criterial recollection tests: Distinctiveness heuristic versus criterion shifts. Journal of Memory \& Language, 51, 473-493. doi:10.1016/j.jml.2004.06.002

Galton, F. (1879). Psychometric experiments. Brain, 2, 149-162.

Garry, M., Manning, C. G., Loftus, E. F., \& Sherman, S. J. (1996) Imagination inflation: Imagining a childhood event inflates confidence that it occurred. Psychonomic Bulletin \& Review, 3, 208-214.

Goff, L. M., \& RoEdiger, H. L., III (1998). Imagination inflation for action events: Repeated imaginings lead to illusory recollections. Memory \& Cognition, 26, 20-33.

Gollwitzer, P. M., \& BrandstätTer, V. (1997). Implementation intentions and effective goal pursuit. Journal of Personality \& Social Psychology, 73, 186-199.

Greenwald, A. G., \& BanaJi, M. R. (1989). The self as a memory system: Powerful, but ordinary. Journal of Personality \& Social Psychology, 57, 41-54.

Gunter, R. W., Bodner, G. E., \& Azad, T. (2007). Generation and mnemonic encoding induce a mirror effect in the DRM paradigm. Memory \& Cognition, 35, 1083-1092.

Hassabis, D., Kumaran, D., \& Maguire, E. A. (2007). Using imagination to understand the neural basis of episodic memory. Journal of Neuroscience, 27, 14365-14374. doi:10.1523/jneurosci.4549-07.2007

Johnson, M. K. (2006). Memory and reality. American Psychologist, 61, 760-771.

Johnson, M. K., Foley, M. A., Suengas, A. G., \& Raye, C. L. (1988). Phenomenal characteristics of memories for perceived and imagined autobiographical events. Journal of Experimental Psychology: General, 117, 371-376.

Johnson, M. K., Hashtroudi, S., \& Lindsay, D. S. (1993). Source monitoring. Psychological Bulletin, 114, 3-28.

Johnson, M. K., \& RAYE, C. L. (1981). Reality monitoring. Psychological Review, 88, 67-85.

Johnson, M. K., RaYe, C. L., Foley, H. J., \& Foley, M. A. (1981). Cognitive operations and decision bias in reality monitoring. American Journal of Psychology, 94, 37-64.

Kealy, K. L. K., \& Arbuthnott, K. D. (2003). Phenomenal characteristics of co-created guided imagery and autobiographical memories. Applied Cognitive Psychology, 17, 801-818. doi:10.1002/acp.910

Lampinen, J. M., Odegard, T. N., \& Bullington, J. L. (2003). Qualities of memories for performed and imagined actions. Applied Cognitive Psychology, 17, 881-893. doi:10.1002/acp.916

Mather, M., HeNKeL, L. A., \& Johnson, M. K. (1997). Evaluating characteristics of false memories: Remember/know judgments and characteristics questionnaire compared. Memory \& Cognition, 25, 826-837.

McDonough, I. M., \& Gallo, D. A. (2008). Autobiographical elaboration reduces memory distortion: Cognitive operations and the distinctiveness heuristic. Journal of Experimental Psychology: Learning, Memory, \& Cognition, 34, 1430-1445. doi:10.1037/a0013013

McGinNis, D., \& RoBERTS, P. (1996). Qualitative characteristics of vivid memories attributed to real and imagined experiences. American Journal of Psychology, 109, 59-77.

Norman, K. A., \& Schacter, D. L. (1997). False recognition in younger and older adults: Exploring the characteristics of illusory memories. Memory \& Cognition, 25, 838-848.

Pezdek, K., Blandon-Gitlin, I., \& Gabbay, P. (2006). Imagination and memory: Does imagining implausible events lead to false autobiographical memories? Psychonomic Bulletin \& Review, 13, 764-769. 
Schacter, D. L., \& AdDIS, D. R. (2007). The cognitive neuroscience of constructive memory: Remembering the past and imagining the future. Philosophical Transactions of the Royal Society, 362, 773-786. doi:10.1098/rstb.2007.2087

Schacter, D. L., AdDis, D. R., \& Buckner, R. L. (2007). Remembering the past to imagine the future: The prospective brain. Nature Reviews Neuroscience, 8, 657-661. doi:10.1038/nrn2213

Schacter, D. L., Israel, L., \& Racine, C. (1999). Suppressing false recognition in younger and older adults: The distinctiveness heuristic. Journal of Memory \& Language, 40, 1-24.

Schacter, D. L., \& Wiseman, A. L. (2006). Reducing memory errors: The distinctiveness heuristic. In R. R. Hunt \& J. B. Worthen (Eds.), Distinctiveness and memory (pp. 89-107). New York: Oxford University Press.

Sharot, T., Riccardi, A. M., Raio, C. M., \& Phelps, E. A. (2007). Neural mechanisms mediating optimism bias. Nature, 450, 102-105. doi: 10.1038 /nature 06280

Spreng, R. N., \& Levine, B. (2006). The temporal distribution of past and future autobiographical events across the life span. Memory \& Cognition, 34, 1644-1651.

Strack, F., \& Bless, H. (1994). Memory for nonoccurrences: Metacognitive and presuppositional strategies. Journal of Memory \& Language, 33, 203-217.

Suddendorf, T., \& Busby, J. (2005). Making decisions with the future in mind: Developmental and comparative identification of mental time travel. Learning \& Motivation, 36, 110-125. doi:10.1016 /j.Imot.2005.02.010
Suddendorf, T., \& Corballis, M. C. (1997). Mental time travel and the evolution of the human mind. Genetic Social \& General Psychology Monographs, 123, 133-167.

Suddendorf, T., \& Corballis, M. C. (2007). The evolution of foresight: What is mental time travel, and is it unique to humans? Behavioral \& Brain Sciences, 30, 299-313. doi:10.1017/S0140525 X07001975

Szpunar, K. K., \& McDermott, K. B. (2008). Episodic future thought and its relation to remembering: Evidence from ratings of subjective experience. Consciousness \& Cognition, 17, 330-334. doi:10.1016 /j.concog.2007.04.006

Szpunar, K. K., Watson, J. M., \& McDermott, K. B. (2007). Neural substrates of envisioning the future. Proceedings of the National Academy of Sciences, 104, 642-647. doi:10.1073/pnas.0610082104

Thomas, A. K., Bulevich, J. B., \& Loftus, E. F. (2003). Exploring the role of repetition and sensory elaboration in the imagination inflation effect. Memory \& Cognition, 31, 630-640.

Tulving, E. (1985). Memory and consciousness. Canadian Psychology, 26, 1-12.

WENZEL, A. (2005). Autobiographical memory tasks in clinical research. In A. Wenzel \& D. C. Rubin (Eds.), Cognitive methods and their applications to clinical research (pp. 243-264). Washington, DC: American Psychological Association.

Wiseman, A. L. (2007). Imagery reduces false recognition in younger and older adults through the use of a distinctiveness heuristic. Dissertation Abstracts International, 67, 7401.

\section{Unique Recollected Detail Instructions}

After deciding whether you retrieved a specific memory [imagined a future event] for the item, you will be asked to make a more specific judgment about your unique recollected details, or distinctiveness, in that memory.

On the recollection detail, or distinctiveness, judgment we would like you to rate the amount of unique details that you can recollect that are different from other items in the study phase, independent of how strong or vivid those recollections may be. Please rate the amount of unique details on a scale of 1 to 7 , where a rating of 1 would indicate that you recollect only a few unique details and a rating of 7 would indicate that you recollect many unique details for the item.

For example, when presented with an item, you may recollect some details that are unique to the item presented. You may remember several different details of the specific memory that you retrieved [imagined future event that you generated] or the process of searching for the specific memory itself [creating the future event itself]; this would be given a rating of 4 or 5 , because the details are unique or distinctive to the item. Alternatively, you may not recollect many details, or you may recollect several details that are not unique to the item presented. Or, to give another example, you may have a general recollection that you retrieved a specific memory for the item (but can no longer remember what that memory was) [that you imagined a future event for the item (but can no longer remember what that event was)] or the memory was from last week (as were many of the other memories) [the future event will occur in one week (as will many of the other events)], but because you have these same sorts of general memories for many of the other items, these recollections would be given a rating of 1 or 2 , because the details are not unique to the specific item presented.

Remember that these judgments are about your memories for distinctive details that you encoded during the study phase, not whether you can think of new unique details during the test. 